COMMENT. Young male patients taking AED therapy for seizures are at risk of significant bone loss at the femoral neck. Almost $50 \%$ have osteopenia, a 2.5 fold increased prevalence compared to the healthy male population, and the risk of fractures is increased.

\title{
LEVETIRACETAM IN PARTIAL SEIZURES
}

The efficacy and safety of levetiracetam (LEV) as adjunctive therapy were evaluated in 23 children (aged 6-12 years) with monotherapy-resistant partialonset seizures at Children's Hospital Medical Center, Cincinnati, OH and other major centers. Seizure frequency during an 8-week evaluation period with individualized LEV doses (20-40 mg/kg/day) was compared with the 4-week baseline seizure frequency. Seizure frequency was reduced by $>50 \%$ in $12(52 \%)$ patients. Plasma concentrations of concomitant AED (carbamazepine or valproic acid) were not affected. Adverse events included headache (33\%), anorexia (25\%), and somnolence (25\%). No alterations in mean clinical laboratory values were observed. A decrease in red blood cell count occurred in 1 patient, and thrombocytopenia in one was attributed to concomitant valproic acid treatment. (Glauser TA, Pellock JM, Bebin EM et al. Efficacy and safety of levetiracetam in children with partial seizures: an open-label trial. Epilepsia May 2002;43:518-524). (Reprints: Dr TA Glauser, Children's Hospital Medical Center, Department of Neurology OSB5, 3333 Burnet Avenue, Cincinnati, OH 45229).

COMMENT. Levetiracetam (Keppra $\left.{ }^{\circledR}\right)$, an adjunctive therapy for partial seizures in adults, is shown to be effective and relatively safe in children, by open-trial. A randomized, placebo-controlled, double-blind trial is ongoing.

In an editorial (Van Ness PC. Therapy for the epilepsies. Arch Neurol May 2002;59:732-733), Levetiracetam, introduced in 1999, is described as a broad spectrum AED, twice-daily dosing, unique mechanism of action, low toxicity, low protein binding, low risk of rash, and no drug interactions. Sedation is the main disadvantage, occurring in $15 \%$ of patients.

\section{DEGENERATIVE DISEASES}

\section{MOLECULAR DIAGNOSIS OF ALEXANDER DISEASE}

The value of clinical and MRI criteria in the diagnosis of Alexander disease was determined by using GFAP gene sequencing as the confirmatory assay in 13 patients with variable ages of onset in a study at Children's National Medical Center, Washington, DC and other centers. Genomic DNA was screened for mutations in the GFAP gene, and $12(92 \%)$ tested positive. Seven of the 12 presented in infancy (ages 2 to 18 nonths) with megalencephaly, seizures, failure to thrive, and delayed development. Five were juvenile-onset (ages 5 to 9 years) with variable symptoms, 2 being asymptomatic and the remainder with severe symptoms, including growth failure, sleepiness, and vomiting. Both groups showed progression of megalencephaly with increasing age, bulbar signs, spasticity, cognitive deficits, and developmental delay. MRI showed diffuse, symmetrical white matter abnormality in the frontal regions, sparing subcortical U-fibers. GFAP gene mutation analysis should be included in the initial diagnostic evaluation of infants or young children presenting with megalencephaly and predominantly frontal leukoencephalopathy on MRI. Gene analysis may now take the place of brain biopsy histological examination for diagnostic Rosenthal fibers. (Gorospe JR, Naidu S, Johnson AB et al. Molecular findings in symptomatic and pre-symptomatic Alexander disease patients. Neurology May (2 of 2) 